\title{
A SOLUTION FORMULA FOR THE STOKES EQUATION IN R\$_+^n\$
}

AUTHOR(S):

Ukai, Seiji

\section{CITATION:}

Ukai, Seiji. A SOLUTION FORMULA FOR THE STOKES EQUATION IN $R \$+{ }^{+\wedge} n$. 数理解析研究所講究録 1987, 604: 124-138

\section{ISSUE DATE:}

1987-01

URL:

http://hdl.handle.net/2433/99666

RIGHT: 
A SOLUTION FORMULA FOR THE STOKES EQUATION IN $\mathbf{R}_{+}^{\mathbf{n}}$

\section{Seiji Ukai (Osaka City University)}

1. Introduction and Main Result

The Stokes equation is the linear equation obtained from the Navier-Stokes equation by ignoring the quadratic convection term. In this paper we discuss its initial boundary value problem in the half space $R_{+}^{n}(n \geq 2)$;

$$
\begin{aligned}
(1.1 a) & u_{t}-\Delta u & +\nabla p=0, \\
(1.1 b) & \nabla \cdot u & =0, \\
(1.1 c) & \gamma u & =a\left(t, x^{\prime}\right), \\
(1.1 d) & \left.u\right|_{t=0} & =u_{o}(x) .
\end{aligned}
$$

Here the unkowns are the velocity $u(t, x)=\left(u^{1}, u^{2}, \ldots, u^{n}\right)$ and the pressure $p(t, x)$ where $t \geq 0$ and $x=\left(x_{1}, x_{2}, \ldots, x_{n}\right)=\left(x^{\prime}, x_{n}\right) \in R^{n-1} \times R_{+}=R_{+}^{n}$, while $\nabla=\left(\partial_{1}, \partial_{2}, \ldots, \partial_{n}\right), \partial_{j}=\partial / \partial x_{j}$, is the gradient, $\Delta$ the laplacian, - means the inner product in $\mathbf{R}^{\mathrm{n}}$ and $\gamma$ is the trace operator to the boundary $\partial R_{+}^{n}=R^{n-1} x\{0\} ; \gamma u=u\left(t, x^{\prime}, 0\right) . \quad a\left(t, x^{\prime}\right)$ and $u_{0}(x)$ are the prescribed boundary and initial values, respectively. In the sequel the tangential components of vectors will be denoted with prime. Thus,

$$
u=\left(u^{\prime}, u^{n}\right), \quad a=\left(a^{\prime}, a^{n}\right), \quad u_{0}=\left(u_{0}^{\prime}, u_{0}^{n}\right) .
$$

The aim of this paper is to explicitly write down the solution to (1.1) in terms of only Riesz' operators and the solution operators for the heat and Laplace's equations in $\mathbf{R}_{+}^{\mathbf{n}}$, all of which are well- 
known operators. Solonikov [7] has already derived such explicit formulas to obtain various estimates of solutions to (1.1). Then his estimates have been used to evaluate solutions to (1.1) for arbitray bounded domains, see e.g. [6,8,9] (see also [4] for a different approach), which is useful to construct strong solutions to the (nonlinear) Navier-Stokes equations, [3]. The formula derived here looks more compact and seems easier to evaluate, and further, our method of derivation is quite different from and simpler than that of Solonikov [7]. Also, the formula (1.10) below which gives the solution to (1.1) for the case $a=0$ is not found in [7] and crucial to construct $L^{p}$-global solutions to the Navier-Stokes equation in $\mathbf{R}_{+}^{\mathrm{n}}$, see section 3 .

We shall introduce two kinds of Riesz' operators, $R_{j}, j=1, \ldots, n$, and $s_{j}, j=1, \ldots, n-1$, which are the singular integral operators with the symbols,

$$
\begin{array}{ll}
\sigma\left(R_{j}\right)=i \xi_{j} /|\xi|, & j=1, \ldots, n, \\
\sigma\left(S_{j}\right)=i \xi_{j} /\left|\xi^{\prime}\right|, & j=1, \ldots, n-1,
\end{array}
$$

where $\xi=\left(\xi_{1}, \xi_{2}, \ldots, \xi_{n}\right)=\left(\xi^{\prime}, \xi_{n}\right) \in R^{n-1} \times R$ is the dual variable to $x \in R^{n}$. Thus $R_{j}$ are defined in the space $R^{n}$ and given explicitly as

$$
R_{j} f(x)=v \cdot p \cdot \int_{R^{n}} R_{j}(x-y) f(y) d y,
$$

where v.p. means the principal part of the integral and

$$
R_{j}(x)=c_{n} x_{j} /|x|^{n+1}, \quad c_{n}=2^{k-n / 2} \sqrt{\pi} \Gamma((n-1) / 2),
$$

$\Gamma$ being the gamma function, and similarly for $s_{j}$ but in $R^{n-1}$. We note that $S_{j}$ can also be considered in $R_{+}^{n}$ as well as in $R^{n}$ in a natural manner. Set 
$(1.2)$

$$
R^{\prime}=\left(R_{1}, R_{2}, \ldots, R_{n-1}\right)
$$$$
s=\left(s_{1}, s_{2}, \ldots, s_{n-1}\right) \text {, }
$$

and define the operators $\mathrm{v}_{1}$ and $\mathrm{v}_{2}$ by

$$
\begin{aligned}
& v_{1} u_{0}=-s \cdot u_{0}^{\prime}+u_{0}^{n}, \\
& v_{2} u_{0}=u_{0}^{\prime}+S u_{0}^{n} .
\end{aligned}
$$

Further, let $r$ be the restriction operator from $\mathbf{R}^{n}$ to $\mathbf{R}_{+}^{n}$, that is,

$$
\text { (1.4a) } \quad \mathrm{rf}=\left.\mathrm{f}\right|_{\mathbf{R}_{+}^{\mathrm{n}}} \text {, }
$$

and $\mathbf{e}$ the extension operator from $\mathbf{R}_{+}^{\mathbf{n}}$ over $\mathbf{R}^{\mathrm{n}}$ with value 0 ;

$(1.4 b) \quad$ ef $=f \quad\left(x_{n}>0\right), \quad=0 \quad\left(x_{n}<0\right)$.

Then we define the operator $U$ by

$$
U f=r R^{\prime} \cdot S\left(R^{\prime} \cdot S+R_{n}\right) e .
$$

We shall further use the heat kernel in the whole space,

$$
E_{O}(t, x)=(4 \pi t)^{-n / 2} \exp \left\{-|x|^{2} /(4 t)\right\}
$$

in order to define the operators $E(t)$ and $F$ respectively by

$$
\left.E(t) f=\int_{R_{+}^{n}}\left\{E_{o}(t, x-y)\right)-E_{o}(t, x+y)\right\} f(y) d y,
$$

$$
F b=\int_{0}^{t} \int_{R^{n-1}} \partial_{n} E_{o}\left(t-s, x^{\prime}-y^{\prime}, x_{n}\right) b\left(s, y^{\prime}\right) d s d y^{\prime},
$$

which are the solution operators to the heat equation in $\mathbf{R}_{+}^{\mathbf{n}}$;

$$
\begin{aligned}
z_{t}-\Delta z & =0, \quad \text { in } R_{+}^{n}, \\
\gamma z & =b\left(t, x^{\prime}\right), \\
\left.z\right|_{t=0} & =z_{0}(x) .
\end{aligned}
$$

Thus $z=E(t) z_{0}$ solves (1.7) for the case $b=0$ while $z=F b$ does for $z_{0}=0$. 
Also, we shall introduce the Poisson operators $D$ and $N$ for the Dirichlet and Neumann problems for Laplace's equation in $\mathbf{R}_{+}^{\mathrm{n}}$, respectively. Thus $z=\mathrm{Db}$ (resp. $z=\mathrm{Nb}$ ) is the unique solution of

$$
\Delta z=0 \text { in } R_{+}^{n},
$$

$$
\gamma z=b\left(x^{\prime}\right) \quad\left(\text { resp. } \gamma \partial_{n} z=b\left(x^{\prime}\right)\right) .
$$

As is well known, they are explicitly given in terms of the single and double layer potentials by

$$
\begin{aligned}
& (1.9 a) \quad \mathrm{Db}=\int_{R^{n-1}} \partial_{n} G\left(x^{\prime}-y^{\prime}, x_{n}\right) b\left(y^{\prime}\right) d y^{\prime}, \\
& (1.9 b) \quad N b=\int_{R^{n-1}} G\left(x^{\prime}-y^{\prime}, x_{n}\right) b\left(y^{\prime}\right) d y^{\prime},
\end{aligned}
$$

where

$$
G(x)=C_{n}|x|^{-(n-2)}(n \geq 3),=-(2 \pi)^{-1} \log |x| \quad(n=2)
$$

is the Newton potential, with $C_{n}=2(n-2)^{-1} \pi^{n / 2} \Gamma(n / 2)$.

Now we are ready to state our solution formulas. For later convinience, they will be given separately for the case $a=0$ and for $u_{0}=0$

Theorem 1.1. Suppose $a=0$. Then the solution to (1.1) can be expressed as

$$
\begin{array}{ll}
(1.10 a) & u^{n}=\operatorname{UE}(t) v_{1} u_{0}, \\
(1.10 b) & u^{\prime}=E(t) v_{2} u_{0}+\operatorname{SUE}(t) v_{1} u_{0}, \\
(1.10 c) & p=-D \gamma \partial_{n} E(t) v_{1} u_{0} .
\end{array}
$$

Theorem 1.2. Suppose $u_{0}=0$. Then the solution to (1.1) is

$$
\begin{aligned}
& (1.11 a) \quad u^{n}=D a^{n}+U F V_{1} a, \\
& (1.11 b) \quad u^{\prime}=F V_{2} a-S\left(D a^{n}+U F V_{1} a\right),
\end{aligned}
$$


$(1.11 c) \quad p=\left|\nabla^{\prime}\right| D V_{1} a+D \gamma \partial_{n} F V_{1} a-N a_{t}^{n}$,

where $\left|\nabla^{\prime}\right|$ is the pseudo differential operator having the symbol $\left|\xi^{\prime}\right|$.

Remak 1.3. (i) Solonikov [7] have not given (1.10) but only (1.11), although in a slightly complex expression.

(ii) In order that these formulas actually give smooth solutions to (1.1), $u_{o}$ and a should not only be smooth but also satisfy the compatibility conditions described in [7]. In particular, $\nabla \cdot u_{0}=0$ is required for $(1.10)$ and $a^{n}(0, x)=0$ for $(1.11)$.

(iii) Evidently, the sum of (1.10) and (1.11) gives the solution to (1.1) when $a \neq 0$ and $u_{0} \neq 0$. However compatibility conditions are required separately for a and $u_{0}$. More resonable is Solonikov' procedure [7]: First we solve the Cauchy problem for (1.1), with $u_{0}$ appropriatly extended over $R^{n}$. Then the solution is $u=E_{o}(t) u_{0}, p=0$, where

$$
E_{0}(t) u_{0}=\int_{R^{n}} E_{0}(t, x-y) u_{0}(y) d y
$$

is just the solution to the Cauchy problem for the heat equation. If we set $u=E_{0}(t) u_{0}+v$ in $(1.1)$, we obtain again (1.1) for $v, p$ with $u_{0}=0$ and a replaced by $a-\gamma E_{0}(t) u_{0}$, to which $(1.11)$ applies. In this case we have compatibility conditions relating a and $u_{0}$, see [7] for details. In case $a=0$, this procedure leads to a different form of the solution from (1.10).

Remark 1.4. Even if $u_{0}$ and a are not smooth nor satisfy compatibility conditions, the above formulas still give solutions to $(1.1)$ in a certain sense. For example, suppose $u_{0} \in L^{p}\left(R_{+}^{n}\right)$ with $1<p<\infty$. Then we can see that for $t>0,(u, p)$ of $(1.10)$ is smooth and satisfies $(1.1 a),(1.1 b)$ and $(1.1 c)$ with $a=0$, and instead of $(1.1 d)$, it holds 
that as $t \downarrow 0$,

$$
u(t) \rightarrow P_{0} u_{o} \quad \text { strongly in } L^{p}\left(R_{+}^{n}\right),
$$

where $P_{0}$ is the operator defined by

$$
\left(P_{0} u_{o}\right)^{\prime}=v_{2} u_{0}-S U V_{1} u_{0},
$$

$$
\left(P_{0} u_{0}\right)^{n}=U V_{1} u_{0} \text {. }
$$

Remark 1.5. It can be shown that if $1<\mathrm{p}<\infty, \mathrm{P}_{\mathrm{O}}$ is a continuous projection from $L^{p}\left(R_{+}^{n}\right)$ onto the solenoidal subspace

$$
P L{ }^{p}\left(R_{+}^{n}\right)=\left\{u_{0} \in L^{P}\left(R_{+}^{n}\right) \mid \nabla \cdot u_{0}=0, r u_{0}^{n}=0\right\} .
$$

Notice, however, that $P_{0}$ does not coincide with the well-known projection $\mathrm{P}$ associated with the Helmholtz decomposition, [2]. In particular, $\mathrm{P}$ is an orthogonal projection but $\mathrm{P}_{\mathrm{o}}$ is not, in the case $p=2$.

\section{Proof of Theorems}

Since we work in the half space $\mathbf{R}_{+}^{\mathbf{n}}$, it is natural to use the Fourier transformation with respect to the tangential variable $x^{\prime}$. Let $f(x)=f\left(x^{\prime}, x_{n}\right)$ be a function defined on $R_{+}^{n}$. Then its Fourier transform in $x^{\prime}$ is defined by

$$
\hat{f}\left(\xi^{\prime}, x_{n}\right)=(2 \pi)^{-(n-1) / 2} \int_{R^{n-1}} e^{i x^{\prime} \cdot \xi^{\prime}} f\left(x^{\prime}, x_{n}\right) d x^{\prime} .
$$

In the sequel we will drop , thus using the same symbol $f$ for both $f$ and its Fourier transform $\hat{f}$, and in accordance with this, we will freely denote the singular integral operator in $x^{\prime}$, say $s$ in (1.2), 
by its symbol, say $\sigma(S)=i \xi^{\prime} /\left|\xi^{\prime}\right|$. This convention will simplifies the notation greatly, not only raise no confusions.

We begin our proof by noting the equation $\Delta p=0$ which comes from (1.1a) and (1.1b). Passing to the Fourier transform in $x^{\prime}$ and recalling our convention, we get the ordinary differential equation

$$
\left(\partial_{n}^{2}-\left|\xi^{\prime}\right|^{2}\right) p=0 \quad \text { in } x_{n}>0
$$

Now we shall solve this assuming that $p$ is bounded. Clearly such solutions must have the form

$$
p=k\left(\xi^{\prime}, x_{n}\right) \gamma p
$$

where

(2.1) $\quad k\left(\xi^{\prime}, s\right)=\exp \left(-\left|\xi^{\prime}\right| s\right)$.

Although the trace $\gamma p$ is still unknown, it follows that $p$ satisfies

$$
\left(\partial_{n}+\left|\xi^{\prime}\right|\right) p=0
$$

This is the key in our argument.

First, we set

$$
z=\left(\partial_{n}+\left|\xi^{\prime}\right|\right) u^{n}
$$

and apply $\left(\partial_{n}+\left|\xi^{\prime}\right|\right)$ to the $n-t h$ equation in (1.1a) to see, by the aid of (2.2), that $z_{t}-\Delta z=0$ holds. Further, since (1.1b) is equivalent to

$$
i \xi^{\prime} \cdot u^{\prime}+\partial_{n} u^{n}=0 \text {, }
$$

we have, together with $(1.1 \mathrm{c})$,

$$
\begin{aligned}
\gamma z & =\gamma\left(\partial_{n}+\left|\xi^{\prime}\right|\right) u^{n}=-i \xi^{\prime} \cdot \gamma u^{\prime}+\left|\xi^{\prime}\right| \gamma u^{n} \\
& =-i \xi^{\prime} \cdot a^{\prime}+\left|\xi^{\prime}\right| a^{n}=\left|\xi^{\prime}\right| V_{1} a
\end{aligned}
$$


and similarly, with $(1.1 d)$,

$$
\left.z\right|_{t=0}=\left|\xi^{\prime}\right| v_{1} u_{0}
$$

where $\mathrm{v}_{1}$ is the operator defined by (1.3). This shows that $z$ defined by (2.3) solves the heat equation (1.6) with $b=\left|\xi^{\prime}\right| V_{1} a$ and $z_{0}=\left|\xi^{\prime}\right| v_{1} u_{0} \cdot$ Accordingly, $z$ is given in the form of

$$
z=\left|\xi^{\prime}\right|\left(E(t) v_{1} u_{0}+F v_{1} a\right)
$$

Note that $\left|\xi^{\prime}\right|=\left|\nabla^{\prime}\right|$ (pseudo differential operator) commutes with $E(t)$ and $\mathrm{F}$.

Now that $z$ is known, $u^{n}$ can be obtained if $(2.3)$ is looked as the ordinary differential equation for $u^{n}$ in $x_{n}>0$ and solved under the boundary condition $\gamma \mathrm{u}^{\mathrm{n}}=\mathrm{a}^{\mathrm{n}}$ which comes from (1.1c). The result is,

$$
u^{n}=k\left(\xi^{\prime}, x_{n}\right) a^{n}+\int_{0}^{x_{n}} k\left(\xi^{\prime}, x_{n}-y_{n}\right) z\left(t, \xi^{\prime}, y_{n}\right) d y_{n},
$$

where $\mathrm{k}$ is as in $(2.1)$.

We shall show that (2.6) coincides with the sum of $(1.10 a)$ and (1.11a). First, we note that the Poisson operator D in (1.8a) has the symbol $\sigma(D)=k\left(\xi^{\prime}, x_{n}\right)$, so the first term on the right hand side of $(2.6)$ is $\mathrm{Da}^{\mathrm{n}}$ (recall our convention). Next, define the operator $\sigma$ by

$$
\sigma f=\left|\xi^{\prime}\right| \int_{0}^{x_{n}} k\left(\xi^{\prime}, x_{n}-y_{n}\right) f\left(\xi^{\prime}, y_{n}\right) d y_{n}
$$

We shall show that this is nothing but $U$ of $(1.5)$. Set $h(s)=$ $\left|\xi^{\prime}\right| k\left(\xi^{\prime}, s\right)$ for $s>0$ and $=0$ for $s<0$. Then we have

$(2.7) . \quad \sigma f=r \int_{-\infty}^{\infty} h\left(x_{n}-y_{n}\right) e f\left(y_{n}\right) d y_{n}$, 
where $r$ and $e$ are as in (1.8). The Fourier transform $h\left(\xi_{n}\right)$ of $h\left(x_{n}\right)$ with rerspect to $x_{n}$ is

$$
\begin{aligned}
h\left(\xi_{n}\right) & =(2 \pi)^{-1 / 2}\left|\xi^{\prime}\right| \int_{0}^{\infty} e^{-\left(\left|\xi^{\prime}\right|+i \xi_{n}\right) x_{n}} d x_{n} \\
& =(2 \pi)^{-1 / 2}\left|\xi^{\prime}\right|\left(\left|\xi^{\prime}\right|+i \xi_{n}\right)^{-1} .
\end{aligned}
$$

Hence we have, recalling $(1.2)$,

$$
\begin{aligned}
(2 \pi)^{1 / 2} \hat{h} & =\left|\xi^{\prime}\right|\left(\left|\xi^{\prime}\right|-i \xi_{n}\right) /|\xi|^{2} \\
& =\sigma(R) \cdot \sigma\left(S^{\prime}\right)\left\{\sigma(R) \cdot \sigma\left(S^{\prime}\right)+\sigma\left(R_{n}\right)\right\} \\
& =\sigma\left(R \cdot S^{\prime}\left(R \cdot S^{\prime}+R_{n}\right)\right) .
\end{aligned}
$$

Since the Fourier transform of the convolution (in $x_{n}$ ) of $h$ and $f$ is $(2 \pi)^{1 / 2} \hat{h f}$, we conclude from $(2.7),(2.8)$ that $\sigma=U$. In view of $(2.5)$, therefore, $(2.6)$ can be rewritten as

$$
u^{n}=D a^{n}+U\left(E(t) v_{1} u_{0}+F v_{1} a\right)
$$

which is what was desired.

To obtain the tangential component $u^{\prime}$, we set

$$
w=v_{2} u=u^{\prime}+S u^{n}=u^{\prime}+\left(i \xi_{n} /\left|\xi^{\prime}\right|\right) u^{n} .
$$

Then it follows from $(1.1 a)$ and (2.2) that

$$
w_{t}-\Delta w=-i \xi^{\prime} p-\left(i \xi^{\prime} /\left|\xi^{\prime}\right|\right) \partial_{n} p=-s\left(\left|\xi^{\prime}\right|+\partial_{n}\right) p=0
$$

and from $(1.1 \mathrm{c})$ and $(1.1 \mathrm{~d})$ that $\gamma w=V_{2} a$ and $\left.w\right|_{t=0}=v_{2} u_{0}$, respectively. Hence we have $w=E(t) V_{2} u_{0}+F V_{2} a$ or

$$
u^{\prime}=E(t) v_{2} u_{0}+F v_{2} a-S u^{n},
$$

which, together with (2.9), gives rise to the sum of $(1.10 \mathrm{~b})$ and $(1.11 \mathrm{~b})$.

It remains to derive the expression for $p$. This is done 
upon substitution of (2.9) into the $n$-th equation of (1.1a);

$$
\partial_{n} p=-\left(u_{t}^{n}-\Delta u^{n}\right)=-D a_{t}^{n}-\left(\partial_{t}-\Delta\right) U\left(E(t) v_{1} u_{0}+F v_{1} a\right)
$$

We note that $\partial_{t} U=U \partial_{t}$ and $\left|\xi^{\prime}\right|^{2} U=U\left|\xi^{\prime}\right|^{2}$ while, since $U=O$ and since $\partial k\left(x_{n}-y_{n}\right) / \partial x_{n}=-\partial k\left(x_{n}-y_{n}\right) / \partial y_{n}$ where $k(s)=k\left(\xi^{\prime}, s\right)$ is that of $(2.1)$, we have by integration by parts,

$$
\begin{aligned}
\partial_{n} U f & =\left|\xi^{\prime}\right| \partial_{n} \int_{0}^{x} k\left(x_{n}-y_{n}, \xi^{\prime}\right) f\left(y_{n}\right) d y_{n} \\
& =\left|\xi^{\prime}\right|\left\{f\left(x_{n}\right)-\left[k\left(x_{n}-y_{n}\right) f\left(y_{n}\right)\right]_{y_{n}=0}^{x_{n}}\right\}+U \partial_{n} f \\
& =\left|\xi^{\prime}\right| k\left(x_{n}\right) \gamma f+U \partial_{n} f .
\end{aligned}
$$

Iterating this, we get

$$
\begin{aligned}
\partial_{n}^{2} U f & =-\left|\xi^{\prime}\right|^{2} k\left(x_{n}\right) \gamma f+\left|\xi^{\prime}\right| k\left(x_{n}\right) \gamma \partial_{n} f+U \partial_{n}^{2} f \\
& =-\left|\xi^{\prime}\right|\left(\left|\xi^{\prime}\right| D \gamma f-D \gamma \partial_{n} f\right)+U \partial_{n}^{2} f
\end{aligned}
$$

Recall that $\sigma(D)=k\left(x_{n}\right)$ for $D$ of $(1.8 a)$. Combining these yields

$$
\left(\partial_{t}-\Delta\right) U f=\left|\xi^{\prime}\right|\left(\left|\xi^{\prime}\right| D \gamma f-D \gamma \partial_{n} f\right)+U\left(\partial_{t}-\Delta\right) f .
$$

Consequently, $(2.10)$ becomes

$$
\begin{aligned}
\partial_{n} p & =-D a_{t}^{n}-\left|\xi^{\prime}\right|\left(\left|\xi^{\prime}\right| D \gamma-D \gamma \partial_{n}\right)\left(E(t) V_{1} u_{o}+F V_{1} a\right) \\
& =\left|\xi^{\prime}\right| D \gamma \partial_{n} E(t) v_{1} u_{0}-D a_{t}^{n}-\left|\xi^{\prime}\right|\left(\left|\xi^{\prime}\right| D V_{1} a+D \gamma \partial_{n} F V_{1} a\right),
\end{aligned}
$$

because $z=E(t) V_{1} u_{0}+F V_{1} a$ solves (1.7) for $b=V_{1}$ a and $z_{0}=v_{1} u_{0}$. But $\partial_{n} p=-\left|\xi^{\prime}\right| p$ by $(2.2)$, so $p$ is given by dividing the last expression in $(2.11)$ by $-\left|\xi^{\prime}\right|$. Noting $-\sigma(D) /\left|\xi^{\prime}\right|=-k\left(x_{n}\right) /\left|\xi^{\prime}\right|=\sigma(N)$, we have

$$
p=-D \gamma \partial_{n} E(t) V_{1} u_{0}+\left|\nabla^{\prime}\right| D V_{1} a+D \gamma \partial_{n} F V_{1} a-N a_{t}^{n} .
$$

This completes the proof of Theorems 1.1 and 1.2 . 
3. $L^{\mathrm{p}}-\mathrm{L}^{\mathrm{q}}$ estimates.

The formulas derived so far provides us with an easy way to evaluate the solution of (1.1) and its derivatives in various function spaces. Here we will illustrate $\mathrm{L}^{\mathrm{p}}-\mathrm{L}^{\mathrm{q}}$ estimates of $\mathrm{u}$ for the case $a=0$, using the formula $(1.10)$. The case $a \neq 0$ can be treated essentially in the same way. In the sequel $\|$. will denote the norm of $L^{p}\left(\mathbf{R}_{+}^{n}\right)$. Our main result is,

Theorem 3.1. Let $u=\left(u^{\prime}, u^{n}\right)$ be the solution to (1.1) for $a=0$. Then, for any $p, q$ with $1<q<p<\infty$, there is a constant $C_{2} 0$ such that

$$
\begin{aligned}
& \|u(t)\|_{p} \leq c t^{-\alpha}\left\|u_{o}\right\|_{q}, \\
& \|\nabla u(t)\|_{p} \leq c t^{-\alpha-1 / 2}\left\|u_{o}\right\|_{q},
\end{aligned}
$$

hold for any $u_{0} \in L^{q}\left(R_{+}^{n}\right)$ and for all $t>0$, with

$$
\alpha=(n / 2)\left(q^{-1}-p^{-1}\right) \text {. }
$$

To prove this we need three lemmas. First, according to Calderón-Zygmund [1], Riesz' operators $R_{j}$ are bounded operators on $L^{p}\left(R^{n}\right)$ and $S_{j}$ on $L^{P}\left(R^{n-1}\right)$, both for $1<p<\infty$. Further, $s_{j}$ can be also looked in a natural way as bounded operators on $L^{p}\left(R_{+}^{n}\right)$ as well as on $L^{p}\left(R^{n}\right)$. Hence we have the

Lemma 3.2. For $1<p<\infty$, the operators $U, V_{1}$ and $V_{2}$ are all bounded on $L^{p}\left(R_{+}^{n}\right)$. Moreover, $V_{1}$ and $V_{2}$ are also bounded on $L^{p}\left(R^{n-1}\right)$ $=L^{P}\left(\partial R_{+}^{n}\right)$.

Next, we recall that $U=U$ where $O$ is defined by (2.8). Putting $k(s)=k\left(\xi^{\prime}, s\right)$ for $(2.1)$ and noting that 


$$
k(0)=1, \quad \partial k(s) / \partial s=-\left|\xi^{\prime}\right| k(s),
$$

we readily see that

$$
\begin{aligned}
\partial_{n} U f & =\left|\xi^{\prime}\right| \partial_{n} \int_{0}^{x_{n}} k\left(x_{n}-y_{n}\right) f\left(y_{n}\right) d y_{n} \\
& =\left|\xi^{\prime}\right|\left\{f\left(x_{n}\right)-U f\left(x_{n}\right)\right\} .
\end{aligned}
$$

Since $\partial_{j}, 1 \leq j \leq n-1$, commute with all $R_{j}$ and $S_{j}$, we thus proved

Lemma 3.3. (i) $a_{j} U=U a_{j}, 1 \leq j \leq n-1$,

(ii) $\quad a_{n} U=(I-U)\left|\nabla^{\prime}\right|$

(iii) $\quad \partial_{j} v_{k}=v_{k} \partial_{j}, \quad 1 \leq j \leq n, \quad k=1,2$

Finally we shall prove that the solution $z(t)=E(t) z_{0}$ to the heat equation (1.7) with $b=0$ enjoys the following $L^{p}-L^{q}$ estimates.

Lemma 3.4 . For $1 \leq q \leq p \leq \infty$, we have

$$
\begin{aligned}
& \left\|E(t) z_{o}\right\|_{p} \leq t^{-\alpha}\left\|z_{o}\right\|_{q}, \\
& \left\|\nabla E(t) z_{o}\right\|_{p} \leq t^{-\alpha-1 / 2}\left\|z_{o}\right\|_{q},
\end{aligned}
$$

for any $z_{0} \in L^{q}\left(R_{+}^{n}\right)$ and $t>0$, with $\alpha$ defined by $(3.3)$.

Proof. Recall $E_{0}(t)$ of $(1.14)$, the solution operator for the heat equation in $R^{n}$. Then $E(t) z_{0}$ defined by $(1.6)$ can be written in terms of $E_{0}(t)$ as

$$
E(t) z_{0}=r E_{0}(t) \widetilde{z}_{0}
$$

where $\widetilde{z}_{0}(x)$ is the odd extention into $x_{n}<0$ of $z_{0}(x)$;

$$
\widetilde{z}_{o}\left(x^{\prime}, x_{n}\right)=z_{o}\left(x^{\prime}, x_{n}\right) \text { for } x_{n}>0 \text {, and }=-z_{o}\left(x^{\prime},-x_{n}\right) \text { for } x_{n}<0 \text {. }
$$

On the other hand, it is well-known from the properties of the heat kernel $E_{o}(t, x)$ that $(3.4)$ and $(3.5)$ holds for $E_{o}(t)$ if the norms are modified for $\mathbf{R}^{\mathrm{n}}$, so that the lemma immediately follows in view of (3.6). 
Proof of Theorem 3.1. Since we are assuming $a=0$, the normal component $u^{n}$ is given by $(1.10 a)$, so we have, using (3.4),

$$
\begin{aligned}
\left\|u^{n}\right\|_{p} & \leq\|U\|_{p}\left\|E(t) v_{1} u_{o}\right\|_{p} \\
& \leq t^{-\alpha}\|U\|_{p}\left\|v_{1} u_{o}\right\|_{q} \\
& \leq t^{-\alpha}\|U\|_{p}\left\|V_{1}\right\|_{q}\left\|u_{o}\right\|_{q},
\end{aligned}
$$

where $\|U\|_{p}$ is the operator norm of $U$ on $L^{p}\left(R_{+}^{n}\right)$ and similarly for $\left\|V_{1}\right\|_{q}$. Since these norms are finite owing to Lemma 3.2 , we have (3.1) for $u^{n}$, and also for $u^{\prime}$, proceeding similarly with (1.10b). The estimate (3.2) can be obtained essentially in the same way if one uses Lemma 3.3 and $(3.5)$, and notices that

$$
\left|\nabla^{\prime}\right|=-\sum_{j=1}^{n-1} s_{j} \partial_{j} .
$$

This completes the proof of Theorem 3.1 .

Remark 3.5. This theorem enables us to construct local and global strong $L^{p}$ solutions to the Navier-Stokes equation in $\mathbf{R}_{+}^{\mathbf{n}}$;

$$
\begin{aligned}
u_{t}+u \cdot \nabla u-\Delta u & +\nabla p=0, \\
\nabla \cdot u & =0, \\
\gamma u & =0, \\
\left.u\right|_{t=0} & =u_{0} .
\end{aligned}
$$

In fact, Giga [3] and Kato [5] proved the existence of such $L^{p}$ solutions for the whole space $\mathbf{R}^{\mathrm{n}}$, and also Giga [3] for arbitrary bounded domains. Their proof makes use of only the estimates (3.1) and (3.2) for the solution of the corresponding Stokes equation. In other words, their proof applies literally to the case $R_{+}^{n}$, by virtue of Theorem 3.1. Thus all theorems in [3,5] remain true for the half 
space $\mathbf{R}_{+}^{\mathrm{n}}$. We will not reproduce them here. As for local $\mathrm{L}^{\mathrm{p}}$ strong solutions in $\mathbf{R}_{+}^{\mathrm{n}}$, see also Weissler [10].

\section{References}

[1] Calderón A.P. and A. zygmund, On singular integrals, Amer. J. Math. 78(1956), 310-320.

[2] Fujiwara, D. and H. Morimoto, An $L_{r}$-theory of the Helmholtz decomposition of vector fields, J. Fac. Sci. Univ. of Tokyo, Sec. 1, 24(1977),685-700.

[3] Giga, Y., Solutions for semi-linear parabolic equations in $L^{p}$ and regularity of weak solutions of the Navier-Stokes system, J. Diff. Equation, 62(1986), 186-212 .

[4] , Analicity of the semi-group generated by the Stokes operator in $\mathrm{L}_{\mathrm{r}}$ spaces, Math. Z. 178(1981), 297-329.

[5] Kato, T., Strong $\mathrm{L}^{\mathrm{p}}$-solutions of the Navier-Stokes equation in $\mathbf{R}^{\mathbf{n}}$ with applications to weak solutions, Math. Z. 187(1984), $471-480$.

[6] Miyakawa,T., On the initial value problem for the Navier-Stokes equations in $\mathrm{L}^{\mathrm{p}}$ spaces, Hiroshima J. Math. 11(1981), 297-329 .

[7] Solonikov, V. A., Estimates of the solutions of a nonstationary linearized system of Navier-Stokes equations, Amer. Math. Soc. Transl. (2), 75(1968), 1-116.

[8] Estimates for solutions of nonstationary Navier-Stokes equations, J. Soviet Math . 8(1977), 685-700.

[9] Wahl, W. von, Regularity questions for the Navier-Stokes 
equations, Approximation Methods for Navier-Stokes Problems, R. Rautmann ed., Lecture Notes in Math. 771, 538-542, Berlin, Heidelberg, New York, Springer, 1980.

[10] Weisseler, F. B., The Navier-Stokes initial value problem in $L^{p}$, Arch. Rational Mech. Anal. 74(1980), 219-230. 